\title{
Clean energy consumption mechanism based on multi-energy transactions with electric heating
}

\author{
Guiyuan XUE ${ }^{1, *}$, Chen $\mathrm{WU}^{1}$,Wenjuan NIU ${ }^{1}$, Xun DOU ${ }^{2}$, Shizhen $\mathrm{WANG}^{2}$ \\ ${ }^{1}$ Economic Research Institute, State Grid Jiangsu Electric Power Co,. LTD, Nanjing, 210000, China \\ ${ }^{2}$ College of Electrical Engineering and Control Science, Nanjing TECH University, Nanjing, 211816, China
}

\begin{abstract}
The comprehensive utilization of multi-energy is an important means to promote the high proportion of clean energy consumption in the region. This paper proposes a clean energy absorption mechanism based on electric heating to participate in multi-energy trading, which is applied to the transaction and operation optimization of energy terminals. In this paper, the scheduling framework of energy terminal is constructed first, and then the optimal scheduling model of energy terminal based on electric heating participating in multi-energy trading is established to promote clean energy consumption through collaborative optimization of multi-energy coupling equipment. The economic dispatch of energy terminal to all kinds of energy is realized. Finally, a test example is constructed to verify the operation economy and clean energy absorption rate of the energy terminal. The simulation shows that the proposed method can not only improve the clean energy absorption rate of the energy terminal, but also improve the operation economy of the system, and verify the effectiveness of the proposed method.
\end{abstract}

\section{Introduction}

Winter heating is the basic livelihood needs of residents in northern China. For a long time, coal-fired centralized heating has been mostly used, which has brought great impact to the environment [1-2]. Among many clean heating methods, electric heating has been strongly supported and promoted by governments and enterprises at all levels because of its advantages of clean environmental protection and strong controllability. On the other hand, with the rapid development of clean energy in recent years, the increasing permeability of clean energy, the volatility and randomness of clean energy consumption and utilization of energy terminals have brought difficulties. Combined with electric heating and other coupling equipment to promote clean energy consumption is of great significance.

At present, scholars at home and abroad have carried out a lot of research on clean energy absorption mechanism. Through the establishment of reasonable power market trading mechanism, the literature [4] put forward the problem of wind abandonment in order to solve the problem of high proportion renewable energy access to power system. Also, the power market trading mechanism can improve the competitiveness of wind power generation in the power market and ensure the complete absorption of wind power. By analyzing the electricity price elasticity of flexible power load [5] the paper establishes an operational model to optimize the period of peak and valley electricity division and the corresponding price level, which ensures the interests of all participants. The peak-valley electricity price system with stronger resource allocation ability is obtained.[6] the literature, the benefit sharing model of cooperative innovation members is studied. The results show that the appropriate transformation mode can maximize the overall and individual benefits. Firstly [7] the typical characteristics of high proportion renewable energy are analyzed, and on this basis, the influence of high proportion renewable energy participation in power market on market mechanism is studied, which can provide reference experience for the construction of power market in China.

To sum up, at present, scholars at home and abroad have some research on promoting clean energy consumption, its research direction is mainly aimed at energy transmission, the optimization of consumption links, and less through the means of energy conversion to promote clean energy consumption. Therefore, it is necessary to use the means of trade to realize the conversion and utilization of electricity, gas and heat energy from the point of view of promoting clean energy consumption, and to improve the clean energy absorption rate of energy terminals.

This paper proposes a clean energy absorption mechanism based on electric heating to participate in multi-energy trading, which is applied to the transaction and operation optimization of energy terminals. Firstly, the scheduling framework of energy terminal is constructed, and then the optimal scheduling model of energy terminal based on electric heating is established. Finally, the simulation results are analyzed to verify the effectiveness of the proposed method. 


\section{Energy flow structure of 1 energy source terminal}

\subsection{Energy terminal energy flow}

The energy terminal energy flow constructed in this paper is shown in figure 1. The external energy sources of the system include END (electric distribution network) and GND (Gas distribution network). A terminal generator includes WT and PV. Coupling equipment include GB (Gas fired boiler), GT (Gas turbine); and EH (Electric heating).



Figure. 1. Energy Terminal Energy Flow

Energy transmission direction: energy terminal can buy electricity and natural gas from energy network. On the one hand, clean energy generation can supply terminal load demand, on the other hand, it can meet terminal heat load demand through electric heating equipment. The gas turbine and gas boiler inside the energy terminal can be used to meet the heat load and electric load demand of the terminal respectively.

\subsection{Clean energy consumption mechanism}

Based on the energy terminal energy flow structure shown in figure 1, a clean energy absorption mechanism based on electric heating to participate in multi-energy trading is constructed. Energy terminal clean energy output first supply terminal load demand. In the peak period of clean energy output, when clean energy output is larger than electricity load demand, electric heating supply heat load is operated to promote clean energy consumption.

\section{Model description}

\subsection{Objective function}

Taking the minimum cost of energy terminal as the objective function, the expression is as follows:

$$
\begin{aligned}
& S=p_{t} \times\left(d z r_{t}+p p_{t}-d e_{t}-p_{g t}\right)+ \\
& p g \times\left(d a g_{t}+q r_{t}+q d_{t}\right)
\end{aligned}
$$

Where, $p_{t}$ and $p g$ are the prices of electricity and gas respectively. $d z r_{t}, p p_{t}, d e_{t}$ and $p_{g t}$ are electric heating power consumption, electric load, clean energy supply and electricity generated by gas boiler, respectively. $\operatorname{dag}_{t}, \quad q r_{t}$ and $q d_{t}$ are gas load, gas power consumption of GT and GB.

The energy conversion formulas for various coupling devices are as follows:

$$
\begin{aligned}
H_{g b} & =\eta_{g b} P_{g b} \\
P_{g t} & =\eta_{g t} P_{g t} \\
H_{E H} & =\eta_{e h} P_{e h}
\end{aligned}
$$

Where, Hgb, Pgt, HEH are gas boiler heat generation power, gas turbine power generation, electric heating heat generation power respectively. $\eta g b$, $\eta g t$, $\eta$ eh are the respective conversion efficiency.

\subsection{Constraints}

(1) Power balance constraints of energy terminals:

$$
m d_{t}=d z r_{t}+p p_{t}-d e_{t}-\eta_{g b} \times q d_{t}
$$

Where, $m d_{t}$ is the purchasing power of energy terminal at time $t$.

(2) Balance constraints of natural gas at energy terminal:

$$
m q_{t}=\operatorname{dag}_{t}+q r_{t}+q d_{t}
$$

Where, dagt is the gas consumption of users at time $t$. qdt, qrt are the gas consumption of GT and GB. mqt is the amount of natural gas purchased from the gas network at time $\mathrm{t}$.

(3) Equilibrium constraints of heat

$$
d a h_{t}=\eta_{e h} \times\left(d z r_{t}+d h_{t}\right)+\eta_{g b} \times q r_{t}
$$

Where, daht is the heat load at time $t$, dht is the amount of clean energy used for heating at time $t$.

(4) Balance constraints of clean energy power generation:

$$
d a w_{t}=d e_{t}+d h_{t}+f q_{t}
$$

Where, dawt is the clean energy generation at time t. fqt is the abandoned air volume at time $t$.

(5) Operational constraints of coupling equipment

The operation constraints of coupling equipment are conventional constraints. For details, please refer to [8].

\section{Example analysis}

\subsection{Basic data}

In order to verify the effectiveness of the proposed method, the simulation analysis is carried out based on the real data of an energy terminal. The energy terminal is directly connected to the power network and the natural gas network. The energy terminal consists of a wind turbine with a capacity of $100 \mathrm{~kW}$ and a photovoltaic generator with a capacity of $100 \mathrm{~kW}$. The total output power curve of wind power and photovoltaic is shown in figure 2. The coupling equipment consists of a $150 \mathrm{KW} \mathrm{GB}$, a $100 \mathrm{~kW}$ GT and a $150 \mathrm{KW} E H$, with energy conversion efficiencies of $0.3,0.5$ and 0.9 respectively. The energy needs of users include electricity, gas and heat, The user's energy demand 
power is shown in figure 2. The price of natural gas remains at $2.98 \mathrm{yuan} / \mathrm{m}^{3}$, and the electricity price information is shown in Figure 3.

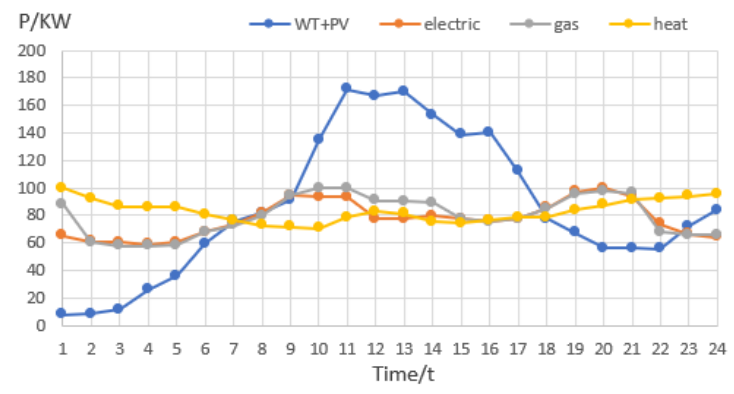

Figure.2. Multi-energy load, clean energy output power

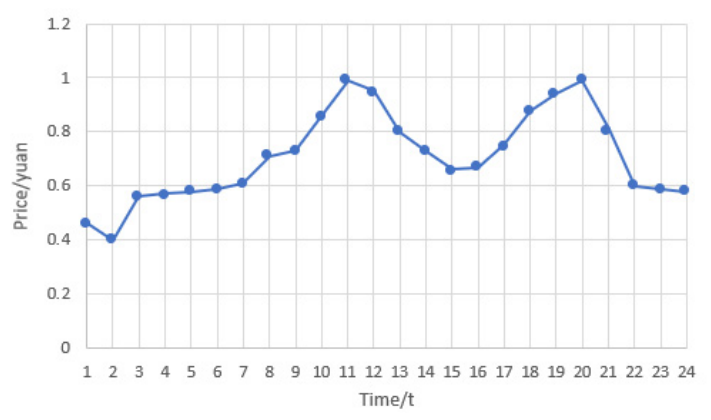

Figure.3. Electricity price curve

\subsection{Result analysis}

(1) Benefit comparison of energy terminal

Through the optimal operation of the proposed method, the purchase of electricity and gas from the main network of the system is shown in Fig.4, in which the total amount of electricity purchased is $1769.74 \mathrm{kwh}$, the total amount of gas purchased is $2259.44 \mathrm{~m}^{3}$. The total cost of purchasing energy is 2116.56 yuan. The amount of electricity purchased was 853 lower $\mathrm{kWh}$, the volume of gas purchased was $326 \mathrm{~m}$ lower than before the optimization3The cost of energy purchase decreased by 1629.5 yuan. It can be seen that through the optimization of clean energy absorption mechanism based on multienergy, it can effectively promote the transformation and utilization of internal resources of energy terminals and reduce energy purchase.

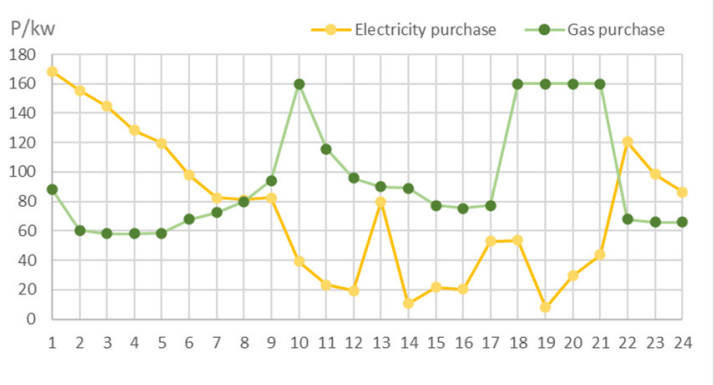

a. Energy purchase curve before optimization



b. Energy purchase curve after optimization

Figure.4. Energy Acquisition Curve of the System before and after Optimization

(2) Comparison of clean energy consumption rates

From figure 4, the curve of clean energy output and the electric load curve of users can be seen that during the peak period of clean energy output at 11-13, the demand for clean energy output will be greater than that of electricity load, and $340 \mathrm{kwh}$ of wind power will be produced every day. Using the mechanism of clean energy absorption based on multi-energy trading proposed in this paper, the clean energy absorption rate is $100 \%$ through the conversion and utilization of coupling equipment between different energy sources. It is proved that the mechanism proposed in this paper is of great significance for the absorption of clean energy.

\section{Conclusion}

Based on multi-energy trading, this paper proposes a clean energy absorption mechanism based on electric heating to participate in multi-energy trading. Firstly, the scheduling framework of an energy terminal is established. Secondly, the optimal scheduling model of energy terminal based on electric heating to participate in multi-energy trading is established. The operating income of energy terminal is optimized. According to the example simulation, the operating economy and clean energy absorption rate of the proposed absorption mechanism are analyzed. The following conclusions can be obtained:

- To promote the conversion and utilization between electric and thermal energy sources by means of transaction, and to improve the economy of the system;

- By considering multi-energy trading and using electric energy with electric heating, it is helpful to clean energy consumption of energy terminal.

- On the basis of multi-energy trading, we will continue to study the impact of various coupling devices on the operation of energy terminals.

\section{Acknowledgments}

This work was supported by Science and technology projects of State Grid Jiangsu Electric Power Design Consulting Co., Ltd. (JE202003).

\section{References}

1. MA Zhiyuan, ZHANG Shining, HOU Fangxin, et al. Exploring the driving factors and their mitigation 
potential in global energy-related $\mathrm{CO} 2$ emission[J]. Global Energy Interconnection, 2020, 3(5): 413-422..

2. ZHOU Weiqing, WU Huacheng, LI Rui, et al. Environment benefit evaluation on UHV transmission in China's air pollution prevention and control action plan[J]. Journal of Global Energy Interconnection, 2018, 1(Supplement 1): 283-289(in Chinese).

3. Feng Yingchun, Li Xuesong, Fan Jie, Jiang Yu. Practice of source network load storage interactive transaction considering clean energy consumption [J]. DSM, 2020,22 (06): 69-74.

4. Luo Jing, sun Weiqing. Study on the trade model of promoting wind energy consumption and energy consumption taking into account demand response [j]. Zhejiang electric power, 2020,39 (12): 50-57.

5. Li Jianhua, Zhou Linggang. Quantitative research on peak valley time sharing pricing strategy for demand response [J]. Zhejiang electric power, 2020,39 (12): 58-64.

6. Gaoshengyu, zhoudongxu, jiaxuefeng, PI Yichen. Benefit sharing mode of power Internet of things Innovation Park driven by collaborative innovation [j] Zhejiang electric power, 2020,39 (12): 65-71.

7. Wang Xin, Pang Jingyu, Peng Shuangwu, Wu Jing. Brazilian electricity market mechanism based on renewable energy and Its Enlightenment to China [J]. Zhejiang electric power, 2020,39 (12): 72-77.

8. Ge Xiaolin, Wang Yunpeng, Zhu Xiaohe, Xia Shu. Day ahead optimal dispatch of electricity heat gas integrated energy system considering differential energy inertia [J / OL]. Power grid technology: 1-13 [2021-03-15] https://doi.org/10.13335/j.10003673.pst.2020.2177. 